\title{
Iris Recognition Using 3D Co-occurrence Matrix
}

\author{
Wen-Shiung $\mathrm{Chen}^{1}$, Ren-Hung Huang ${ }^{1}$, and Lili Hsieh ${ }^{2}$ \\ ${ }^{1}$ VIP-CCLab., Dept. of Electrical Engineering, National Chi Nan University, Taiwan \\ ${ }^{2}$ Dept. of Information Management, Hsiuping Institute of Technology, Taiwan
}

\begin{abstract}
This paper presents a biometric recognition based on the iris of a human eye using gray-level co-occurrence matrix (GLCM). A new approach of GLCM, called 3D-GLCM, which is expanded from the original 2D-GLCM is proposed and used to extract the iris features. The experimental results show that the proposed approach gains an encouraging performance on the UBIRIS iris database. The recognition rate up to $99.65 \%$ can be achieved.
\end{abstract}

Keywords: Personal Authentication, Biometrics, Iris Recognition, Co-occurrence Matrix.

\section{Introduction}

Biometrics [1] refers to automatic identity authentication of a person on a basis of one's unique physiological or behavioral characteristics and is inherently more suitable for discriminating between an authorized person and an impostor than traditional methods. To date, many biometric features, including fingerprint, hand geometry or palmprint, face, and iris [2]-[10], have been studied. In recent years, the human iris keeps receiving growing interest due to its high uniqueness, high permanence, and high circumvention.

The iris, a kind of physiological biometric feature with genetic independence, contains extremely information-rich physical structure and unique texture patterns, and thus is highly complex enough to be used as a biometric signature [2]. Statistical analysis of faker and authentic scores reveals that irises have an exceptionally high degree-of-freedom up to 266 (much more than fingerprint, showing about 78) [2], and thus are the most mathematically unique feature of the human body. The highly randomized appearance of the iris makes its use as a biometric well recognized.

Compared with other biometric features such as hand and fingerprint, iris patterns are more stable and reliable. Each person's irises are unique, and they are stable with age [2]. In addition, several studies have shown that normal variations in coloring and structure of the tissues of an iris are so multitudinous that no two irises are ever alike not even our own or identical twins [2]. As a result, the human iris promises to deliver a high level of uniqueness to authentication applications that other biometrics cannot match. Furthermore, iris recognition systems can be non-invasive to their end-users. In this paper, we investigate and design an automatic biometric iris recognition system using the three-dimensional gray level co-occurrence matrix (3D-GLCM) for the discriminating texture features. 


\section{Related Work}

Nowadays, iris has a high potential in becoming the focus of a very new biometric means of authentication. In 1993, Daugman developed a successful system by using the 2D Gabor wavelet transform [2]. In this system, the visible texture of a person's iris in a real-time video image is encoded into a compact sequence of multi-scale quadrature 2D Gabor wavelet coefficients, which most significant bits consist of a 256-byte "iris code." In 1996, Wildes et al. developed a prototype system based on an automatic iris recognition that uses a very computationally demanding image registration technique [3]. This system exploits normalized correlation over small tiles within the Laplacian pyramid bands as a goodness of match measure. Boles and Boashash [4] proposed an iris identification in which zero-crossing of the wavelet transform at various resolution levels is calculated over concentric circles on the iris, and the resulting 1D signals are compared with the model features using different dissimilarity functions. Zhu et al. [5] proposed an algorithm for global iris texture feature extraction using multi-channel Gabor filtering and wavelet transform. The mean and standard deviation of each sub-image at different resolution levels are extracted as the features. Subsequently, Ma et al. [6] modified the work in [5] by using a bank of circular symmetric filters to extract much more local texture information. The work in [7] also used wavelet multi-resolution analysis based on Gabor filtering for iris feature extraction. Lim et al. [8] used wavelet transform to make a feature vector compact and efficient. In summary, all of the previous studies are based upon the multi-resolution analysis technique. Recently, Monro et al. [9] proposed a DCT-based iris recognition that the differences of DCT coefficients of overlapped angular patches from normalized iris images are encoded. Thornton et al. [10] presented a Bayesian framework for iris pattern matching that experience in-plane nonlinear deformations.

The original gray level co-occurrence matrix (GLCM) [11] proposed by Haralick et al. in 1973, one of the most known texture analysis methods, estimates image properties related to second-order statistics. It is based on counting the order of cooccurrence of brightness value of pixels pairs at a certain distance and a direction, and successfully applied to some works in image processing and biometrics. The texture analysis based on the GLCM method was proposed to measure the texture features created from a natural image for classification [12], segmentation [13] and retrieval [14]. The GLCM technique has also applied to biometrics, such as fingerprint [15], face [16], signature [17] and iris [18]-[21]. The GLCM methods used in iris recognition are two-dimensional. In this paper, a novel 3D approach modified from $2 \mathrm{D}$ version was designed and then applied to extract the iris features more efficiently.

\section{System Overview and Pre-processing}

\subsection{System Overview}

The proposed framework consists of three modules: image pre-processing, feature extraction, and recognition modules, as shown in Fig. 1. First, the iris image preprocessing (IIP) module employs some image processing algorithms to demarcate the region of interest (i.e., iris zone) from the input image containing an eye. It performs three major tasks including iris localization, iris segmentation and coordinate 
transformation, and enhancement for the input iris image. Next, the feature extraction (IFE) module produces a newly designed three-dimensional gray level co-occurrence matrix (3D-GLCM), gathers static properties features, and applies appropriate coding methods on these features to generate the iris feature code. Finally, the minimum distance classifier according to Euclidean distance metric is employed in the iris pattern recognition (IPR) module.

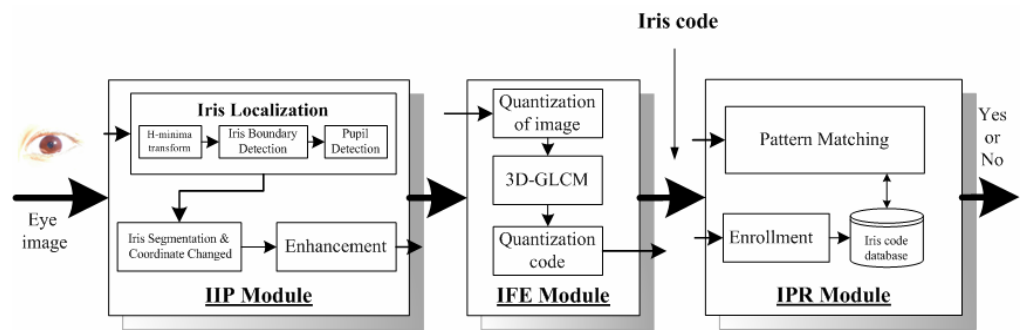

Fig. 1. Structure diagram of the proposed iris recognition system

\subsection{Pre-processing Module}

Input image does contain not only useful information from iris zone but also useless data derived from the surrounding eye region. Before extracting the features of an iris, the input image must be pre-processed to localize, segment and enhance the region of interest (i.e., iris zone). The system normalizes the iris region to overcome the problem of a change in camera-to-eye distance and pupil's size variation derived from illumination. Furthermore, the brightness is not uniformly distributed due to nonuniform illumination; the system must be capable of removing the effect and further enhancing the iris image for the following feature extraction. Hence, the image preprocessing module is composed of three units: iris localization, iris segmentation and coordinate transform, and enhancement units, as shown in Fig. 2.

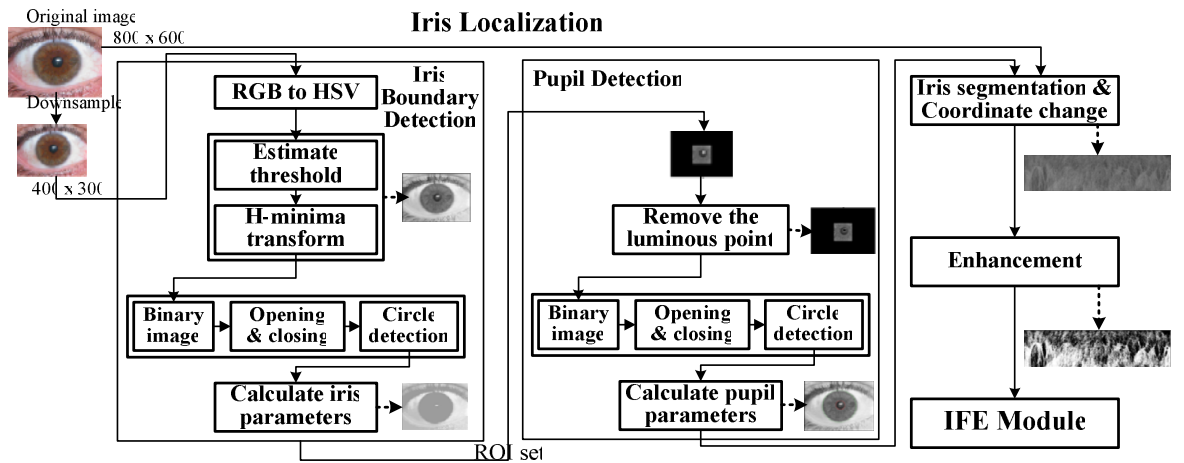

Fig. 2. Pre-processing module 


\section{A. Iris Localization Unit}

In this unit, we must first determine the useful part (i.e., iris) from an input image. The iris is an annular region between the pupil and the sclera. The inner and outer boundaries of an iris can be treated as non-concentric circles approximately. In order to make the iris localization efficient, the system performs an operation of enhancing principal edges and blurring useless edges on the copied and down-sample image instead of the original one. Following that, the system estimates the center coordinates of the iris first. Since the iris typically is darker than the Sclera and its gray level distribution has a small variance, the system uses Extended-Minima (EM) morphology operator [23]. EM transform is the regional minima of the H-minima transform. HMinima transform suppresses all minima in the intensity image which depth is less than a scalar. Regional minima are connected with components of pixels with the same intensity value which external boundary pixels all have a greater value than a scalar. We use 8-connected neighborhoods in this process. By choosing an appropriate scalar in EM transform, a perfect edge of outer boundary is gotten. The value of threshold is decided according to the histogram.

Because of inside of iris of pupil, we use a radius 1.2 times as large as outer circle radius to set a ROI area. We regard it as the pupil and search the range (see Fig. 4(a)). Since the pupil typically is darker than the iris and its gray level distribution has a small variance, the system uses a $30 \times 30$ mean filter (see Fig. 4(d)) to find the location which has the minimum mean value in the image. This location is considered as an internal point of the iris region, namely the fitting point. Then the system can determine a certain region starting from the fitting point using the iris localization procedure. Since there are obvious intensity differences around each boundary, a proper edge detection method can be applied to find the edge points in this region, and evaluate the exact circle parameters (including the inner and outer boundaries of iris) for all possible point triplets of the edge points.

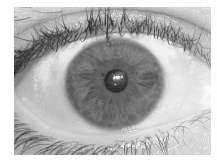

(a)

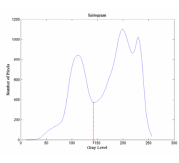

(b)

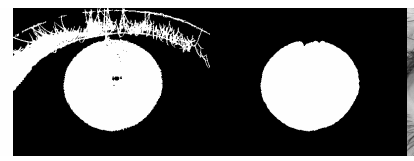

(c) (d)

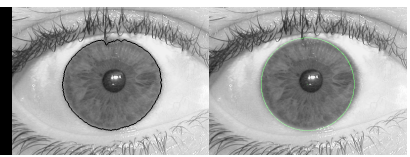

(e)

Fig. 3. (a) Original image, (b) image histogram, (c) EM transform, (d) exact outer edge, (e) iris edge and (f) outer localized image

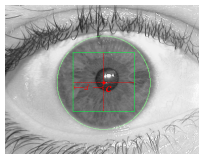

(a)

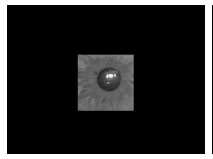

(b)

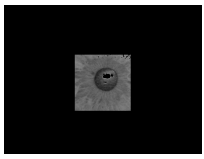

(c)

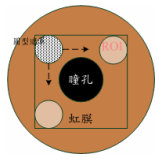

(d)

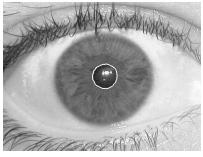

(e)

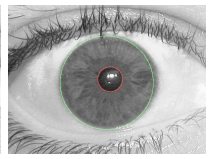

(f)

Fig. 4. (a) Set ROI. (b) ROI image. (c) Remove the luminous point. (d) $30 \times 30$ mean filter. (e) Exact inner edge. (f) Iris localized image. 
According to Euclidean geometry, by calculating two perpendicular bisectors of any edge point triplet, the system can easily obtain the crossover point of two perpendicular bisectors. We select the center point as the most frequently crossed point. After finding the central points of two circles, we determine the inner radius and the outer radius of the iris by computing the distances between the central points and the inner edge points, and outer edge points, respectively. Eventually, an iris zone can be located by these parameters, as shown in Fig. 4(f).

\section{B. Iris Segmentation and Coordinate Transform Unit}

The localized iris zone demarcated from the human eye is transformed from rectangular into polar coordinate systems so as to facilitate the following feature extraction module. When acquiring the human eye image, the iris zone images may be in different sizes (outer and inner boundaries of iris) due to the variations of camera-to-eye distance and/or environmental illumination. The variations will change the patterns of iris texture to some extent. To solve this problem, it is necessary to compensate for the iris deformation. Daugman's system uses radial scaling to compensate for overall size as well as a simple model of pupil variation based on linear stretching [2]. This scaling serves to map Cartesian image coordinates to dimensionless polar image coordinates. In addition, eyelids and eyelashes generally obscure the upper limbus of the iris, so the procedure needs to cut out the obscured area. The system generates a rectangular iris image of a fixed size by linear interpolation. The size of iris image is $512 \times 128$.

\section{The Proposed Method}

\subsection{Two-Dimensional Gray Level Co-occurrence Matrix (2D-GLCM)}

Co-occurrence matrix, proposed by Haralick et al. in 1973 [11], is based on counting the order of co-occurrence of brightness value of pixels pairs at certain distance and direction, which can be described in the following equation:

$$
P_{\text {COM }}(i, j, d, \theta)=N\left\langle\begin{array}{l|l}
\left(\left(x_{n 1}, y_{n 1}\right),\left(x_{n 2}, y_{n 2}\right)\right) \in M_{1} \times M_{2} & \begin{array}{l}
\max \left(\left|x_{n 1}-x_{n 2}\right|,\left|y_{n 1}-y_{n 2}\right|\right)=d \\
\Theta\left(\left(x_{n 1}, y_{n 1}\right),\left(x_{n 2}, y_{n 2}\right)\right)=\theta \\
I\left(x_{n 1}, y_{n 1}\right)=i, I\left(x_{n 2}, y_{n 2}\right)=j
\end{array}
\end{array}\right\rangle
$$

where $\theta$ and $d$ are the direction and distance between two pixels in images, $N$ is the number of elements in the set, and $I$ is an image with the dimension $M_{1} \times M_{2}$ and the range of brightness value $N$ of the processing space. The direction $\theta$ is quantized to four directions: horizontal, diagonal, vertical, and anti-diagonal (corresponding to $0^{\circ}$, $45^{\circ}, 90^{\circ}$ and $135^{\circ}$, respectively). Traditionally, Haralick's feature vectors [11] are computed from the co-occurrence matrices. Specifically, the features of CON, HOM, $A S M, H$, and $C O R$ were usually used for texture analysis.

\subsection{Three-Dimensional Gray Level Co-occurrence Matrix (3D-GLCM)}

\section{A. 3D-GLCM Generation}

The edge structures in iris images are often the most important features for pattern recognition and clear at a variety of scales. To capture the spatial details of an image, 
it is advantageous to make use of co-occurrence matrix. Since the 2D-GLCM is unable to fully represent the texture features of the space domain images, we propose a new approach of GLCM, called 3D-GLCM, which is expanded from the original 2DGLCM and thus can strengthen and demonstrate the texture features of the space domain images. It can be described in the following equation:

$$
P_{C O M}(i, j, k, d, \theta)=N\left(\begin{array}{l|l}
\left(\left(x_{n 1}, y_{n 1}\right),\left(x_{n 2}, y_{n 2}\right),\left(x_{n 3}, y_{n 3}\right)\right) \in M_{1} \times M_{2} \times M_{3} & \begin{array}{l}
\max \left(\left|x_{n 1}-x_{n 2}\right|,\left|y_{n 1}-y_{n 2}\right|\right)=d \\
\max \left(\left|x_{n 2}-x_{n 3}\right|,\left|y_{n 2}-y_{n 3}\right|\right)=d \\
\Theta\left(\left(x_{n 1}, y_{n n}\right),\left(x_{n 3}, y_{n 3}\right)\right)=\theta \\
I\left(x_{n 1}, y_{n 1}\right)=i \\
I\left(x_{n 2}, y_{n 2}\right)=j \\
I\left(x_{n 3}, y_{n 3}\right)=k
\end{array}
\end{array}\right\}
$$

Fig. 5(a) shows the spatial pixel relation where $R$ is the referenced pixel, $N_{1}$ is the 1 st neighbour, and $\mathrm{N}_{2}$ is the 2nd. The structure of 3D-GLCM is shown in Fig. 5(b).

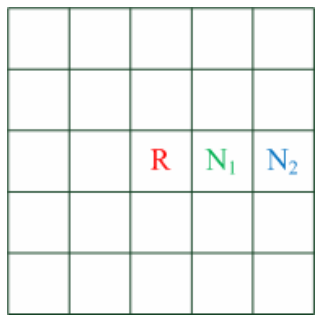

(a)

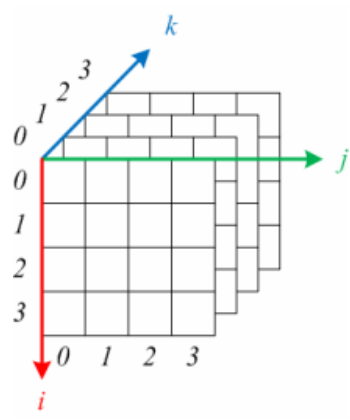

(b)

Fig. 5. (a) Spatial pixel relation. (b) Structure of 3D-GLCM.

\section{B. Feature Extraction using 3D-GLCM Feature Indexes}

By modifying the original 2D-GLCM feature indexes, we design the corresponding new 3D-GLCM feature indexes. These new mathematical operators are listed below.

\begin{tabular}{|l|l|l|}
\hline \multicolumn{1}{|c|}{ Features } & \multicolumn{1}{|c|}{ Definitions } & \\
\hline \hline contrast & $C O N=\sum_{i, j, k=0}^{Q-1} P_{i, j, k}\left[(i-j)^{2}+(i-k)^{2}+(j-k)^{2}\right]$ & $(3)$ \\
\hline homogeneity & $H O M=\sum_{i, j, k=0}^{Q-1} \frac{P_{i, j, k}}{1+\left[(i-j)^{2}+(i-k)^{2}+(j-k)^{2}\right]}$ & (4) \\
\hline angular second moment & $A S M=\sum_{i, j, k=0}^{Q-1} P_{i, j, k}^{2}$ & $(5)$ \\
\hline entropy & $H=\sum_{i, j, k=0}^{Q-1} P_{i, j, k}\left(-\ln P_{i, j, k}\right)$ & $(6)$ \\
\hline image pixel correlation & $C O R=\sum_{i, j, k=0}^{Q-1} P_{i, j, k}\left[\frac{\left(i-\mu_{i}\right)\left(j-\mu_{j}\right)\left(k-\mu_{k}\right)}{\sigma_{i} \sigma_{j} \sigma_{k}}\right]$ & $(7)$ \\
\hline
\end{tabular}




\section{Feature Extraction via Projection}

Since we cannot obtain good recognition results by using the 3D-GLCM feature indexes to compute the features of texture, we introduced the projection method in which the $3 \mathrm{D}$ co-occurrence matrix is projected to three $2 \mathrm{D}$ planes for computing the feature vectors. The proposed 3D-GLCM projection method consists of several steps, as shown in Fig. 6. First, we segment the iris image into $n$ blocks and compute 3D-GLCM for each of the image blocks. Next, we project the 3D-GLCM into three 2D planes and compute the feature vectors from each of these $2 \mathrm{D}$ planes using the $2 \mathrm{D}$ feature indexes.

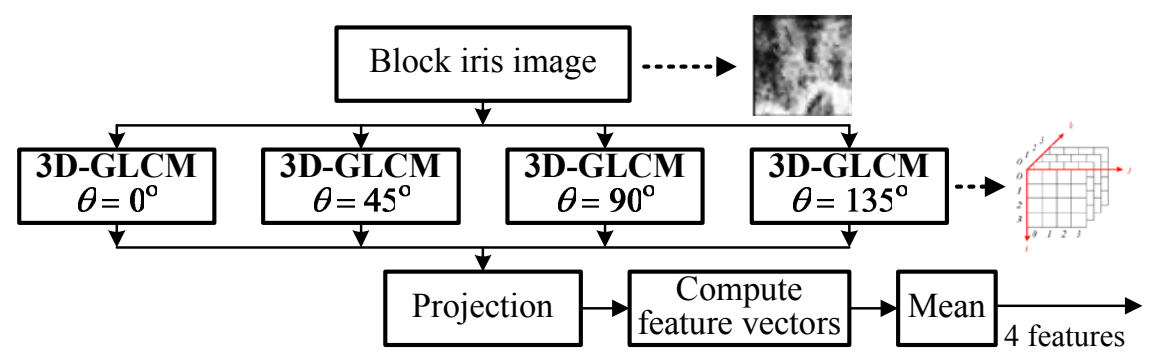

Fig. 6. Feature extraction

\subsection{Pattern Recognition Module}

In this module, the feature code vector extracted from the claimant iris image is compared against those of the enrolled feature code vectors in our iris database. Here for simplicity, we adopt the mean vector as the prototype of each pattern class in the enrollment phase and utilize the minimum distance classifier to check the approach in the recognition phase. When the feature code vector is compared, we calculate the normalized Euclidean distance $D$ of feature vectors $g_{1}$ and $g_{2}$, which is defined as

$$
D\left(g_{1}, g_{2}\right)=\frac{1}{255 \cdot N} \min _{\text {rad }}\left(\sum_{m=1}^{N}\left(g_{1}(m+r a d)-g_{2}(m)\right)^{2}\right)^{\frac{1}{2}}, \quad D \in[0,1]
$$

where $m$ denotes the position of restructured signal and $\mathrm{rad}$ correction of the rotation effect of the input image. Both minimum distance classifiers are normalized to $[0,1]$.

\section{Experimental Results}

To evaluate the performance of the proposed human iris recognition system, we implemented and tested the proposed schemes on the UBIRIS iris image database [22]. The database comprises 1,205 iris images captured from 241 different eyes (hence, 241 classes).

\subsection{Results of Image Pre-processing}

We check the accuracy of the boundaries (including pupil, iris, and lower eyelid) subjectively and the proposed system obtains the success rate of $87.22 \%(1,051$ 
images) from 1,205 images in the experiments for the pre-processing module. It is worth noting that the two main causes of failure come from occlusion by eyelids and non-uniform illumination. Among those 1,051 images obtained successfully from the image pre-processing module, we select 975 images (195 classes) out of them for testing (enrollment and recognition). We train the system by selecting 3 images as the training images set for each person from the authorized users in the enrollment phase. Hence, there are 390 images for testing.

\subsection{Results of the 2D-GLCM Method}

In this experiment, we test the recognition performance for the 2D-GLCM. To obtain a threshold for separating FRR and FAR, we perform two tests: one is for false rejection test and the other is for false acceptance rate test. For the case of FRR, we can obtain the distribution of non-matching distance between the unknown classes and the registered classes. For the case of FAR, we also obtain the distribution of non-matching between the unknown classes for impostors and the registered classes. Fig. 7(a) shows the distributions of the above two experiments. In this figure, the $x$-axis and $y$-axis indicate the degree of distance and the number of data, respectively. Fig. 7(b) shows the plot of the variation of FRR and FAR according to the distribution of non-matching distance by selecting a proper distance threshold. When we set the threshold to be 0.1117 , the system obtains the recognition performance of about $E E R=1.55 \%$. And when the FAR is set to be $0 \%$, the system can obtain FRR $=21.79 \%$ at a threshold of 0.04. As a result of the bad performance of FAR, we adopt our proposed approaches to improve further.

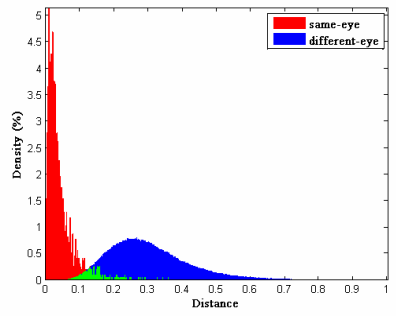

(a)

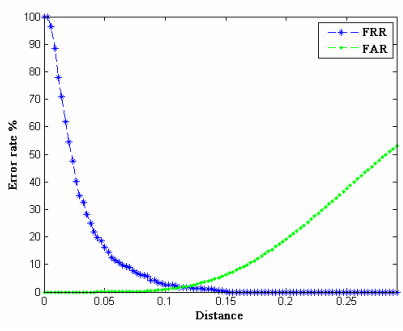

(b)

Fig. 7. The results of the 2D-GLCM method

\subsection{Results of the 3D-GLCM Method}

In this experiment, we test the performance for the 3D-GLCM method. The distribution of non-matching distance for FRR and FAR experiments is shown in Fig. 8(a). Fig. 8(b) shows the plot of the variation of FRR and FAR in the DM method by selecting a proper distance threshold. By selecting the threshold of 0.1217 , the system obtains the system performance of EER $=1.13 \%$. Similarly, if the FAR is set to be $0 \%$, the system can obtain $\mathrm{FRR}=8.72 \%$ at a threshold of 0.061 . In particular, if we use a code vector of 192 bytes instead of 64 bytes, the recognition performance of the proposed system will be reduced to $0.35 \%$ only. The experimental results show that the proposed system performs well. 


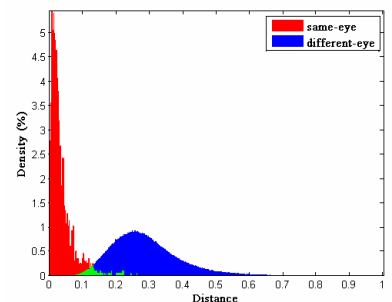

(a)

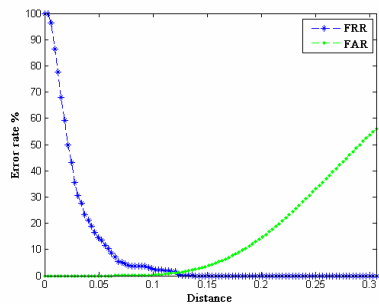

(b)

Fig. 8. The results of the 3D-GLCM method

Finally, we make a summary of the experimental results in Table 1 . We may observe that 3D-GLCM methods have more superior performance than the 2D-GLCM method in the results. On the other hand, the 3D-GLCM method can perform superiorly in the case of $\mathrm{FAR}=0 \%$. Consequently, the 3D-GLCM method provides a securer system than the 2D-GLCM methods.

Table 1. Identification accuracy of the GDC, DM, and CFDM methods

\begin{tabular}{|c|c|c|c|c|c|c|}
\hline Mode & IFE Module & Size of Features & RA $(\boldsymbol{\%})$ & AA $(\%)$ & AF $(\%)$ & RF (\%) \\
\hline \hline \multirow{5}{*}{ EER } & Projection & 256 bytes & 0.51 & 99.49 & 0.51 & 99.49 \\
\cline { 2 - 7 } & Projection & 192 bytes & 0.35 & 99.65 & 0.35 & 99.65 \\
\cline { 2 - 7 } & Projection & 128 bytes & 0.59 & 99.41 & 0.59 & 99.41 \\
\cline { 2 - 7 } & Projection & 64 bytes & 1.13 & 98.87 & 1.13 & 98.87 \\
\cline { 2 - 7 } & 3D-GLCM & 64 bytes & 5.17 & 94.83 & 5.17 & 94.83 \\
\cline { 2 - 7 } & 2D-GLCM & 128 bytes & 1.28 & 98.72 & 1.28 & 98.72 \\
\cline { 2 - 7 } & 2D-GLCM & 64 bytes & 1.55 & 98.45 & 1.55 & 98.45 \\
\hline \hline \multirow{5}{*}{ FAR = \% } & Projection & 256 bytes & 5.38 & 94.62 & 0 & 100 \\
\cline { 2 - 7 } & Projection & 192 bytes & 5.64 & 94.36 & 0 & 100 \\
\cline { 2 - 7 } & Projection & 128 bytes & 8.97 & 91.03 & 0 & 100 \\
\cline { 2 - 7 } & Projection & 64 bytes & 8.72 & 91.28 & 0 & 100 \\
\cline { 2 - 7 } & 3D-GLCM & 64 bytes & 90.77 & 9.23 & 0 & 100 \\
\cline { 2 - 7 } & 2D-GLCM & 128 bytes & 16.15 & 83.85 & 0 & 100 \\
\cline { 2 - 7 } & 2D-GLCM & 64 bytes & 21.79 & 78.21 & 0 & 100 \\
\hline
\end{tabular}

\section{Conclusion}

In this paper, a personal identification with iris recognition using three-dimensional gray level co-occurrence matrix has been proposed. We use two different methods, including 2D-GLCM and 3D-GLCM, in the feature extraction. The 3D-GLCM method can obtain a good recognition rate. The experimental result shows that the proposed iris recognition is suitable for the environment at high security level. 


\section{References}

1. Miller, B.: Vital Signs of Identity. IEEE Spectrum 31, 22-30 (1994)

2. Daugman, J.G.: High Confidence Visual Recognition of Persons by a Test of Statistical Independence. IEEE Trans. Pattern Analysis and Machine Intell. 15, 1148-1161 (1993)

3. Wildes, R.P., et al.: A machine-Vision System for Iris Recognition. Machine Vision and Applications 9, 1-8 (1996)

4. Boles, W.W., Boashash, B.: A Human Identification Technique using Images of the Iris and Wavelet Transform. IEEE Trans. on Signal Processing 46, 1185-1188 (1998)

5. Zhu, Y., Tan, T., Wang, Y.: Biometric Personal Identification based on Iris Patterns. In: Proc. of Int. Conf. on Pattern Recognition, vol. II, pp. 801-804 (2000)

6. Ma, L., Wang, Y., Tan, T.: Iris Recognition using Circular Symmetric Filters. In: Proc. of Int. Conf. on Pattern Recognition, vol. II, pp. 414-417 (2002)

7. Ma, L., Tan, T., Wang, Y., Zhang, D.: Personal Identification based on Iris Texture Analysis. IEEE Trans. on Pattern Analysis and Machine Intelligence 25, 1519-1533 (2003)

8. Lim, S., Lee, K., Byeon, O., Kim, T.: Efficient Iris Recognition Through Improvement of Feature Vector and Classifier. ETRI Journal 23, 61-70 (2001)

9. Monro, D., Rakshit, M.S., Zhang, D.: DCT-based Iris Recognition. IEEE Trans. on Pattern Analysis and Machine Intelligence 29, 586-595 (2007)

10. Thornton, J., Savvides, M., Vijaya Kumar, B.V.K.: A Bayesian Approach to Deformed Pattern Matching of Iris Images. IEEE Trans. on Pattern Analysis and Machine Intelligence 29, 596-606 (2007)

11. Haralick, R.M., Shanmugam, K., Dinstein, L.: Textural Features for Image Classification. IEEE Trans. on Systems, Man, and Cybernetics 3, 610-621 (1973)

12. Valkealahti, K., Oja, E.: Reduced Multidimensional Co-occurrence Histograms in Texture Classification. IEEE Trans. on Pattern Analysis and Machine Intelligence 20, 90-94 (1998)

13. Zwiggelaar, R.: Texture based Segmentation: Automatic Selection of Co-occurrence Matrices. In: 17th Int. Conf. on Pattern Recognition, vol. 1, pp. 588-591 (2004)

14. Partio, M., Cramariuc, B., Gabbouj, M., Visa, A.: Rock Texture Retrieval using Gray Level Cco-occurrence Matrix. In: 6th Nordic Signal Processing Symposium, Norway (2002)

15. Yazdi, M., et al.: Novel Ridge Orientation based Approach for Fingerprint Identification using Co-occurrence Matrix. In: Proc. of World Academy of Science, Engineering and Technology, vol. 26, pp. 371-375 (2007)

16. Dabbah, M.A., Woo, W.L., Dlay, S.S.: Secure Authentication for Face Recognition. In: Proc. of IEEE Symp. on Comput. Intell. In: Image and Signal Proc., pp. 121-126 (2007)

17. Busch, A., et al.: Texture for Script Identification. IEEE Trans. on Pattern Analysis and Machine Intelligence 27, 1720-1732 (2005)

18. Szewczyk, R., et al.: Automatic People Identification on the Basis of Iris Pattern - extraction features and classification. In: Proc. Int. Conf. on Microelectronics, pp. 691-694 (2002)

19. Gupta, G., Agarwal, M.: Iris Recognition using Non Filter-based Techniques. In: Biometric Consortium Conference (2005)

20. Bachoo, A.K., Tapamo, J.R.: Texture Analysis and Unsupervised Clustering for Segmenting Iris Images. In: ACM Int. Conf. Proc. Series, vol. 150, pp. 236-243 (2005)

21. Zaim, A., et al.: A New Method for Iris Recognition using Gray-Level Co-occurrence Matrix. In: IEEE International Conference on Electro/information Technology, pp. 350-353 (2006)

22. Proenc, H., Alexandre, L.A.: Ubiris Iris Image Database, http://iris.di . ubi .pt

23. Poursaberi, A., Araabi, B.N.: A Novel Iris Recognition System using Morphological Edge Detector and Wavelet Phase Features. ICGST Int. J. on Graphics, Vision and Image Processing 5, 9-15 (2005) 